\title{
TEEKAP: Self-Expiring Data Capsule using Trusted Execution Environment
}

\author{
Mingyuan Gao \\ mingyuan.gao@u.nus.edu \\ National University of Singapore \\ Singapore
}

\author{
Hung Dang* \\ hungdang@irics.vn \\ IRICS \\ Vietnam
}

\author{
Ee-Chien Chang \\ changec@comp.nus.edu.sg \\ National University of Singapore \\ Singapore
}

\begin{abstract}
Safeguarding privacy in data sharing is challenging, especially when data owners lose control over their data once it is passed to another party. Our work aims to build a data-sharing platform that enables data owners to regain control over their shared data. Specifically, sensitive data is first encapsulated into a data capsule. The platform regulates functional access to the data capsule, i.e., the receiver can compute a predefined function on the data with its input and learns nothing else. The platform also enforces self-expiry of the data capsule. In addition, the data capsule features a notion of "sendand-forget" wherein data owners can go offline after releasing their data capsules. As a result, data capsules can be freely circulated.

Each data capsule is associated with an access policy and a usage transcript. The former specifies which functions are eligible to access the protected data and its expiry conditions, whereas the latter is used to determine if the expiry conditions have been met. To regulate functional access, one efficient solution is to employ a Trusted Execution Environment (TEE) with attested execution. Nonetheless, we observe that the use of TEE alone is not sufficient to accomplish self-expiry, for TEEs are vulnerable to rollback attacks via which an adversary could "unwind" the usage transcript of an expired data capsule or double-consume the protected data. Moreover, a straightforward implementation would need a single master key to be resided in the TEE, leading to single-point-of-failure. Our solution, TEEKAP, addresses the challenges by embracing decentralization, employing a committee of independent and mutually distrusting nodes to uphold the integrity of usage transcripts and the confidentiality of encryption keys. TEEKAP integrates TEE, consensus protocol, and threshold secret sharing in a novel way. Experiments conducted in realistic deployment settings on Microsoft Azure show that TEEKAP can process access requests at scale.
\end{abstract}

\section{CCS CONCEPTS}

- Security and privacy $\rightarrow$ Access control; Hardware-based security protocols; Privacy protections.

\section{KEYWORDS}

data-sharing platform, data privacy, self-expiry, Intel SGX

\footnotetext{
*This work was done while the author was with National University of Singapore.
}

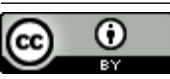

This work is licensed under a Creative Commons Attribution International 4.0 License.

ACSAC '21, December 6-10, 2021, Virtual Event, USA

(c) 2021 Copyright held by the owner/author(s)

ACM ISBN 978-1-4503-8579-4/21/12.

https://doi.org/10.1145/3485832.3485919
ACM Reference Format:

Mingyuan Gao, Hung Dang, and Ee-Chien Chang. 2021. TEEKAP: SelfExpiring Data Capsule using Trusted Execution Environment. In Annual Computer Security Applications Conference (ACSAC '21), December 6-10, 2021, Virtual Event, USA. ACM, New York, NY, USA, 13 pages. https://doi.org/10. $1145 / 3485832.3485919$

\section{INTRODUCTION}

In this era of information explosion, data sharing is undoubtedly a necessity. Nonetheless, the risk of sensitive data being exposed to unintended parties and the lack of technical means to prevent such hazard could dissuade data owners from sharing their data. This concern is ever more relevant in the wake of recent devastating data breach incidents, which were reported to have far-reaching and serious implications at both individual and institutional levels [25, 35, 36]. Protecting data privacy, nonetheless, faces with various daunting challenges. The chief reason lies in the fact that data owners lose control over their data once it is passed to another party. For instance, in the context of cloud services, personal and sensitive data are typically collected, stored, processed and archived by third parties, whose trustworthiness are questionable at times (e.g., Facebook data scandal [25]).

Our work aims to build a data-sharing platform that enables data owners to regain control over their shared data. Specifically, the data owner encapsulates her sensitive data into a data capsule. The platform regulates functional access to the data capsule, i.e., a data capsule user can compute a predefined function on the data with its input and learns nothing else. The platform also enforces self-expiry of the data capsule. Once the expiry conditions for a data capsule are met, the sensitive data stored inside becomes inaccessbile permanently. In addition, the data capsule features a notion of "send-and-forget" wherein data owners can go offline after releasing their data capsules. As a result, data capsules can be freely circulated, or deposited into a public storage.

Each data capsule is associated with an access policy set forth by the data owner and a usage transcript that records all functional accesses performed on the sensitive data thus far. The former specifies which functions are eligible to access the sensitive data and expiry conditions for the sensitive data, whereas the latter keeps track of the access history which is used to determine if the expiry conditions have been met. Access to the sensitive data, however, is subject to both the access policy and its expiry status. When the expiry conditions are met, the sensitive data is rendered unattainable permanently without any involvement from the data owner or parties that store or cache the data capsule.

Data expiry, based on date and usage, is of significant importance, especially when the data capsule itself is circulated unrestrictedly. In many circumstances, the data being shared is only relevant during 
a short period of time following the act of sharing. It is desired that such data becomes inaccessible after the period of time has passed, as guided by the principle of least privilege and a notion of the "right to be forgotten" in GDPR [45]. Examples include disappering messages in WhatsApp [29], stories in Facebook [19], and contact tracing data in COVID-19 responses [2]. Besides, as output of each functional access may reveal some sensitive information of the protected data, it is important to limit access to mitigate probing attacks. For instance, various privacy protection techniques, such as Differential Privacy [18], pose a privacy budget on the protected data (e.g., based on the number of times that access to the data can be granted). Once that budget is exhausted, access to the protected data should no longer be granted, otherwise the privacy protection guarantees degrade. In such techniques, probing attacks can be considered as exhausting the privacy budget.

To regulate functional access, one can ideally utilize functional encryption [5], but this scheme does not have any practical implementations yet. One efficient solution is to leverage a Trusted Execution Environment (TEE) with attested execution [20, 43]. Nonetheless, we observe that the use of TEE alone is not sufficient to accomplish self-expiry, for TEEs are vulnerable to rollback attacks [7, 32] that can fool the TEE into accepting stale data. Via such attacks, an adversary could "unwind" the usage transcript of an expired data capsule or double-consume the protected data. In addition, some straightforward implementations might employ a single master key which is resided in the TEE of every participating entity. Although the use of this single master key would greatly simplify the system design, the breach of a single TEE would lead to failure of the whole system. While many accept the security gurantees provided by TEEs, there are still concerns that individual TEEs could be compromised. Hence, we have to consider single-point-offailure in our design. That is, compromise of any participating TEE could collapse the whole system.

This paper presents TEEKAP ${ }^{1}$, a platform for self-expiring data encapsulation, that addresses the above challenges. TEEKAP employs an access control committee, consisting of independent and mutually distrusting nodes, to collectively maintain two important pieces of information associated with a data capsule, namely, usage transcript and encryption key. On one hand, the committee ensures integrity of the usage transcript even in the presence of individual node failures (e.g., node crash) and guards them against rollback attacks that attempt to circumvent a data capsule's self-expiry. On the other hand, on behalf of data owners, the committee manages the encryption key for each data capsule in a confidential way that is robust against single-point-of-failure issues. To this end, each node in the committee is provisioned a TEE that is associated with a private memory, and runs the access control logic inside such private memory. We remark that it is possible to have a committee consisting of non-TEE powered nodes. Nonetheless, the use of TEE enhances the security and fault tolerance of the committee against malicious entities [3].

Specifically, the data owner encapsulates her sensitive data Data by first encrypting it using a randomly generated encryption key $\mathrm{k}$ (the resulting ciphertext is part of the data capsule). She then "splits" k into shares using a threshold secret-sharing scheme [31,

\footnotetext{
${ }^{1}$ Kápsoula dedoménon means data capsule in Greek.
}

40]. Subsequently, she deposits the key shares and access policy (i.e., which function $\mathcal{F}$ can access Data, and expiry conditions for Data) to nodes in the committee. The nodes rely on a consensus protocol to ensure their view of the usage transcript for a data capsule converge, thereby ensuring its integrity and validity. Any party that wishes to access the protected data Data using a function $\mathcal{F}$ must first provision a TEE that houses $\mathcal{F}$ (referred to as the $\mathcal{F}$ TEE). He then attests the correct instantiation of the $\mathcal{F}$ TEE to the committee [1]. The committee collectively verify the eligibility of $\mathcal{F}$ based on the corresponding access policy, and check the expiry status of the requested data capsule based on its usage transcript. If the access can be granted, nodes in the committee securely deliver the key shares to the $\mathcal{F}$ TEE, wherein the decryption key is reconstructed from a subset of the key shares [31, 40]. If the expiry conditions are met after this approval, the nodes collectively discard the associated key shares.

We built a prototype of TEEKAP using Intel SGX [33] and evaluated its performance on Microsoft Azure using SGX-enabled virtual machines. Experimental results show that an access control committee spanning over separate geographical regions can process access requests at scale.

In summary, this work makes the following contributions.

- We propose and formulate the problem of self-expiring data encapsulation that supports functional access to the protected data and generic user-defined expiry conditions in an autonomous manner.

- We identify two important pieces of information necessitated in implementing self-expiring data capsules, and the technical challenges in guarding them against attacks.

- We present our solution, TEEKAP, that addresses the technical challenges and attains the desired goals. TEEKAP integrates TEE, consensus protocol, and threshold secret sharing in a novel way.

- We build a prototype of TEEKAP, conduct experiments in realistic deployment settings, and empirically show the efficiency of our solution. Our prototype is publicly available at https://github.com/TEELinker/TEEKAP.

\section{PRELIMINARIES}

In this section, we give an overview of the building blocks that we employ in the design of TEEKAP. Section 2.1 presents salient features of Intel SGX, Section 2.2 describes the basics of consensus protocols, and Section 2.3 introduces threshold secret sharing.

\subsection{Intel SGX}

Enclave Execution. While there exist various hardware primitives that can provision TEEs (e.g., Intel SGX [33], Sanctum [15] or Keystone [27]), our work employs Intel SGX due to its widespread availability. Intel SGX is a set of CPU extensions designed to provide hardware-protected TEE (or enclave) for generic computation. Intel SGX associates each enclave process with a CPU-guarded address space (aka enclave memory). The CPU prevents any foreign process (i.e., non-enclave process) from accessing the enclave memory. The enclave is isolated from other enclaves concurrently running on the same host, from the OS, and from other user processes. 
Attestation. Intel SGX provides attestation mechanisms [1] that enable a validator to verify if an enclave in question is instantiated with the correct code. At the same time, these mechanisms provide means via which the validator and the attesting enclave can establish a secure and authenticated channel to communicate sensitive or private data.

If the validator is another enclave instantiated on the same platform (or host) with the attesting enclave, it can use local attestation to ascertain the correct instantiation of the latter. Specifically, after the attesting enclave is initiated, the processor produces an enclave report (i.e., the hash of its initial state), and then creates a message authentication code (MAC) of such measurement using a key that can only be retrieved by the validating enclave. The measurement of the attesting enclave and its MAC are sent to the validating enclave for verification.

On the other hand, if the validator is a remote party, a remote attestation will be performed. In particular, the processor signs the enclave report with its attestation key, generating an enclave quote. In current SGX architecture, the remote party obtaining the enclave quote has to rely on an attestation service for Intel $S G X^{2}$ to check if the signature contained in the enclave quote is valid. Once the signature is validated, the validator checks the values in the enclave quote against known values to make certain of the correct instantiation of the attesting enclave.

Data Sealing. Enclave memory is volatile. To persist their private state to non-volatile storage, the enclave first obtains a unique key that is bound to its measurement from the processor. Subsequently, it encrypts the data using the enclave-specific key, and offloads the encrypted data to the storage. This data sealing mechanism guarantees that the sealed data can only be retrieved by the enclave that sealed it. However, data sealing and retrieving is susceptible to rollback attacks $[7,32]$ wherein an adversary (e.g., the malicious OS) presents the resuming enclave with properly sealed but stale data. We refer readers to $[7,32]$ for details on rollback attacks.

\subsection{Consensus Protocols}

In a distributed system comprising of independent and mutually distrusting nodes, having the nodes agree on some data that is crucial for the operation of the system is challenging, even more so in the presence of node failures. There are two types of node failures, namely crash failure and Byzantine failure. The former characterizes situations wherein a node abruptly stops and does not resume [38], while the latter observes a faulty node deviating arbitrarily from its expected behavior. For instance, a node experiencing Byzantine failure may equivocate, i.e., sending contradictory messages to other nodes, or it may intentionally delay its activity for any period of time [9].

Consensus protocols are designed to achieve safety and liveness in the presence of node failures. Safety necessitates non-faulty nodes to reach an agreement on a value and never return conflicting results for the same query, whereas liveness requires that these nodes eventually agree on a value. The rest of this section focuses on a particular crash-failure consensus protocol called Raft [38].

\footnotetext{
${ }^{2}$ Intel SGX currently supports two types of remote attestation [12]: the Intel Attestation Service for SGX (IAS) [13] based on Intel Enhanced Privacy ID (EPID), and third-party attestation services based on the Elliptic Curve Digital Signature Algorithm (ECDSA).
}

Raft Consensus Protocol. Raft assumes a system of $n$ deterministic processes (or nodes), among which at most $f=\left\lfloor\frac{n-1}{2}\right\rfloor$ could be faulty. Each process stores a log that contains a series of commands, or events of interest. Raft ensures that logs of non-faulty processes contain the same sequence of commands, thereby achieving safety even in asynchronous network (i.e., regardless of timing), and necessarily depends on timing to offer liveness [21] (e.g., network is partially synchronous such that messages are delivered within an unknown but finite bound).

In Raft, each node assumes one of the three roles, namely leader, follower and candidate, and time is split into terms numbered by consecutive integers. Each term marks one node as the leader, while other nodes are followers. The leader maintains its authority by periodically exchanging heartbeats with all followers. Should a follower fail to hear from the leader after an election timeout period, it shall consider the leader crashed, increment its own term, undertake the candidate role and trigger the leader election. The candidate claims itself as a leader once it has collected votes from a majority of nodes. More detailed discussion on the leader election and its election criteria can be found in the Raft paper [38].

Followers respond to requests from the leader and candidate, and stay passive otherwise. All commands (e.g., clients' requests) are sent to the leader, and subsequently replicated on the followers. Given a command, the leader first appends it as a new entry uniquely identified by the leader's term and an index to its log. It then announces the entry to all followers. Upon receiving a new entry, followers append it to their logs, and acknowledge the receipt with the leader. After receiving acknowledgements from a majority of nodes in the system (i.e., $f+1$ or more nodes), the leader commits the entry by executing the command it contains, as well as all preceding entries in its log if they have not been committed. The leader keeps track of the highest index committed, and conveys this information to the followers in subsequent messages so as to inform the latter on the committed entries.

\subsection{Threshold Secret Sharing}

A threshold secret-sharing scheme $[31,40]$ preserves a secret $S$ in a distributed manner. It is parameterized by two values, namely $n$ and $t$, wherein $0<t \leq n$. Specifically, the secret $S$ is split into $n$ shares, and any subset of $t$ or more shares can be used to reconstruct the original secret $S$, wherein the value of $t$ is called the threshold. The security of the scheme dictates that no adversary that possesses knowledge of any $t-1$ or fewer shares could determine or reveal any information about $S$.

Shamir's Secret Sharing [40] works as follows. Given a secret $S$, the threshold $t$ and the number of shares $n$, wherein $t, n$ and $S$ are elements in a finite field $F$ of size $P, 0<t \leq n<P, S<P$, and $P$ is a prime number, one can secret-share $S$ as follows. First, let $a_{0}=S$, and choose $t-1$ positive integers $a_{1}, \ldots, a_{t-1}$ at random such that $a_{i}<P \forall i \in[1, t-1]$. Next, construct a polynomial $f(x)=a_{0}+a_{1} x+a_{2} x^{2}+\ldots+a_{t-1} x^{t-1}$, and pick $n$ points out of form $(j, f(j))$ on $f(x)$ 's graph. Every such point (plus the knowledge of the prime number $P$ defining the finite field $F$ ) constitutes a share. Given the knowledge of any $t$ or more such points, one can evaluate the coefficients $a_{0}, a_{1}, \ldots, a_{t-1}$ of $f(x)$ using interpolation, and obtain the secret $S$ from the constant term $a_{0}$. 


\section{THE PROBLEM}

In this section, we describe the system goals, security requirements and technical challenges in designing self-expiring data capsule systems. Section 3.1 presents the definition of such a system and its constituents, whereas Section 3.2 describes its system goals and security requirements. Section 3.3 provides a running example to illustrate the items defined and specified above. Section 3.4 describes the technical challenges of such a system.

\subsection{Problem Defininition}

A data owner Owner wishes to encapsulate her sensitive data Data into a data capsule $C$. We define a data capsule system and its constituents as follows:

- Data Capsule C: It consists of the ciphertext of Data, and some metadata like ID and the encryption algorithm used to produce the ciphertext. Here, the ciphertext is produced with a randomly generated encryption key $\mathrm{k}$ under a symmetric encryption scheme [26]. Each $C$ can be distributed to its intended receivers. Each $C$ is associated with an access policy $\mathcal{P}$ and a usage transcript $\mathcal{T}$.

- Usage Transcript $\mathcal{T}$ : It chronicles the lifecycle of $C$. This includes the creation of $C$ and accesses that have been granted on $C$. In the basic form, the usage transcript is a counter whose value is the number of times that $C$ has been accessed.

- Access Policy $\mathcal{P}$ : It comprises two pieces of information. First, it specifies which function $\mathcal{F}$ can access Data. We assume that a function $\mathcal{F}$ can be precisely represented by its measurement $M_{\mathcal{F}}$ which is a 256-bit hash identifying the code and the correct instantiation of $\mathcal{F}$ [33]. We abuse the notation to denote the fact that $\mathcal{F}$ is eligible to access Data by $M_{\mathcal{F}} \in \mathcal{P}$. Second, $\mathcal{P}$ contains the threshold values for the expiry conditions. $C$ expires as soon as $\mathcal{T}$ reaches the threshold values contained in $\mathcal{P}$. In the basic form, the access policy consists of the measurement $M_{\mathcal{F}}$ of the authorised function $\mathcal{F}$ and a threshold value specifying the total number of times Data can be accessed.

\subsection{System Goals and Security Requirements}

We first enumerate the goals and features that a self-expiring data capsule system must offer:

(G1) Secure Functional Access: Access to C's Data is granted only to a specific function $\mathcal{F}$. An authorized requester with an input $X$ can compute $\mathcal{F}($ Data, $X)$ but nothing else. In other words, the requester is unable to extract other information from Data except the value of $\mathcal{F}($ Data, $X)$.

(G2) Access Control and Data Expiry: Given a data capsule $C$, its associated access policy $\mathcal{P}$ and usage transcript $\mathcal{T}$, and a function $\mathcal{F}$ with measurement $M_{\mathcal{F}}, \mathcal{F}$ is granted access to $C$ 's Data if and only if $M_{\mathcal{F}} \in \mathcal{P}$ and $\operatorname{Is} \operatorname{ExpiRed}(\mathcal{T}, \mathcal{P})$ returns false; here, the predicate IsExPIRED can determine if $C$ has been expired based on its $\mathcal{T}$ and $\mathcal{P}$. Note that once $\mathcal{C}$ expires, access is denied, even by a function $\mathcal{F}$ with $M_{\mathcal{F}} \in \mathcal{P}$ (i.e., $\mathcal{F}$ was previously able to access Data prior to $C$ 's expiry) ${ }^{3}$.

\footnotetext{
${ }^{3}$ This requires that $\mathcal{F}$ does not persist nor leak Data or any key material that enables it to decapsulate $C$ to obtain Data during its processing of Data.
}

Self-expiry of $C$ is similar to the "right to be forgotten" for personal data in GDPR [45].

(G3) Send-and-Forget: Once Data has been encapsulated into a data capsule $C$, access control and expiry of $C$ should be performed in an autonomous manner, without explicit involvement from its data owner. The data capsule $C$ can be freely circulated, or deposited into a public storage.

To attain goal G1, the system needs to ensure that Data is consumed in a confidential way. To attain goal $G 2$, the system additionally needs to uphold the integrity of $\mathcal{T}$ and prevents Data from double-consumption. To attain all the goals, the system needs to uphold the following three implied security invariants:

(I1) Confidentiality of Data. Recall that the data capsule $C$ is generated by first encrypting the sensitive data Data under a randomly generated encryption key k. Provided that the encryption scheme in use is secure, no party can reveal any information about Data from $C$ without the knowledge of $\mathrm{k}$. Thus, to ensure the confidentiality of Data against unauthorised parties, it suffices to keep $\mathrm{k}$ private. It can only be revealed to the eligible function once the access is granted.

(I2) Integrity of $\mathcal{T}$. Since the expiry status of $C$ is determined by $\mathcal{T}$ and $\mathcal{P}$ (which is fixed after $C$ 's creation), it is critical to ensure that $\mathcal{T}$ always reflects the updated usage status of $C$. Furthermore, it should be guarded against tampers or falsifications in the presence of adversaries.

(I3) Defense Against Double-Consumption of Data. Via the doubleconsumption attack, an adversary may attempt to compute on C's Data multiple times even though the usage transcript $\mathcal{T}$ records a single access to $C$. This violates the access control criteria, especially expiry, of the data capsule $C$. Therefore, the system needs to prevent the adversary from successfully double-consuming C's Data. To facilitate analysis, a more precise definition is required.

Definition 1 (Double-Consumption of Data). Let $r$ be a request for a single functional access of $\mathcal{F}$ on $C$ 's Data, and $\mathcal{T}$ be the usage transcript associated with $C$ that records an access granting to $r$. Once $r$ is permitted, it allows the access requester with input $X$ to compute $\mathcal{F}($ Data, $X)$. A double-consumption of Data occurs if the adversary manages to compute both $\mathcal{F}\left(\right.$ Data, $\left.X_{1}\right)$ and $\mathcal{F}\left(\right.$ Data, $\left.X_{2}\right)$ wherein $X_{1} \neq X_{2}$ with a single $r$ recorded in $\mathcal{T}$.

\subsection{Running Example}

We use the following running example to illustrate our definition of the self-expiring data capsule system and its constituents, as well as the system goals and security requirements. Let us consider an online dating application that matches users based on their profiles, which are likely to contain personal and sensitive information such as users' age and preferences. For simplicity, we assume that the application runs on mobile devices, and relies on a function $\mathcal{F}$ to measure the matching scores between users' profiles. Due to privacy concerns, users do not want to make their profiles public. Consequently, the application keeps the user's profile locally on her mobile device, and encrypts the profile before uploading it to the application's storage server. In order to perform matches, the application running on user $A$ 's mobile device fetches encrypted 
profiles of other users from the storage server, computes the matching scores between those profiles and that of $A$ using $\mathcal{F}$, and notifies $A$ of a match if the matching score is higher than some threshold. Besides, the users have an option to set the limit on which their profiles are matched against, and would like their profiles rendered inaccessible once such a limit is reached.

In this running example, each user is a data owner Owner, and her profile is the sensitive data Data. Each user may encrypt her profile with a unique, randomly generated encryption key $\mathrm{k}$ to obtain the data capsule $C$ 's ciphertext. Since the users are mutually distrusted, they do not want to share the keys with one another. The encrypted profile can be freely circulated, or stored on a public storage. Only the matching function $\mathcal{F}$ can access the users' profiles in their plaintext form. Since each user may set different limits on her profile, each data capsule is subject to a different expiry condition (which in this case is the number of times the profile can be matched against). To prevent the adversary from deviating $\mathcal{F}$ from its intended execution logic and thereby leaking sensitive information contained in the users' profiles, the execution of $\mathcal{F}$ must be carried out exclusively inside a protected environment, for instance a TEE with attested execution. Besides, since users are not involved in the matching procedure, their profiles should be handled in an autonomous manner by the dating application's platform, without leaking sensitve information of the profiles to any unauthorised parties.

\subsection{Strawman Solution and Challenges}

Designing a self-expiring data capsule system that attains all the goals and features presented in Section 3.2 is technically challenging. Although TEEs with attested execution can be employed to regulate functional access (i.e., evaluating and enforcing the access control criteria, as well as ensuring the authorised functions, once granted the key to decapsulate the data capsule, do not deviate from their intended logic or misuse the key), its usage alone is not sufficient to accompolish self-expiry of the data capsule. For sake of clarity, we present a strawman solution, and analyse its weaknesses.

Strawman Solution. In a strawman solution, one can provision the access control functionality inside a TEE, called the Checker TEE. It features attested execution and maintains the encryption key $\mathrm{k}$ associated with the data capsule $C$ inside TEE's private memory ${ }^{4}$ It is also responsible for maintaining the usage transcript $\mathcal{T}$.

The function $\mathcal{F}$ that consumes Data is to be executed in a TEE with attested execution, called the $\mathcal{F}$ TEE. In this approach, the $\mathcal{F}$ TEE must attest its correct instantiation to the Checker TEE. The attestation procedure between the two TEEs also facilitates establishing a secure and authenticated communication channel [1] If $\mathcal{F}$ is eligible to access $C$ and $C$ has not been expired, the Checker TEE sends $\mathrm{k}$ to the $\mathcal{F}$ TEE via this channel. After obtaining $\mathrm{k}$, the $\mathcal{F}$ TEE decapsulates $C$ and consumes Data inside its private memory, thereby preserving Data's confidentiality. Once the computation completes, the $\mathcal{F}$ TEE discards Data and $k$, outputting only $\mathcal{F}($ Data, $X)$ for some $X$ provided by the access requester.

\footnotetext{
${ }^{4} \mathrm{k}$ can be sealed to an external persistent storage in such a way that it can only be retrieved by the Checker TEE [33].
}

Challenges. This strawman solution, unfortunately, admits various weaknesses in upkeeping the Checker TEE, and ignores the rollback and replay attacks on the $\mathcal{F}$ TEE.

First, rollback attacks may cause the Checker TEE to use an obsolete $\mathcal{T}$, thereby failing to enforce expiry of $C$. Second, if confidentiality protection of the Checker TEE is compromised, all encryption keys are leaked. One may battle the rollback attacks against the Checker TEE using the replicated state machine approach [39], i.e., employing a committee of independent nodes to collectively implement the functionality of access control. Specifically, the usage transcript $\mathcal{T}$ is replicated across all the nodes in the committee, and determining whether the corresponding data capsule $C$ has been expired requires a consensus among the nodes. This approach strengthens the integrity protection of $\mathcal{T}$, for launching a coordinated rollback attack on a set of independent nodes is arguably much harder than perpetrating such an attack on a single node (refer to Section 6.3 for its argument). Nonetheless, care must be taken to assure confidentiality of $\mathrm{k}$, since merely replicating the key does not mitigate the threats posed by attacks against confidentiality of TEE execution. As soon as the adversary successfully extracts $k$ from one of the nodes, the security of the system collapses.

What's worse, rollback and replay attacks perpetrated on the $\mathcal{F}$ TEE could enable the adversary to double-consume Data, i.e., computing on Data multiple times even though it is given a single access to Data. Concretely, let us consider two inputs $X_{1}$ and $X_{2}$. The adversary wants to compute both $\mathcal{F}\left(\right.$ Data, $\left.X_{1}\right)$ and $\mathcal{F}\left(\right.$ Data, $\left.X_{2}\right)$, which constitutes two separate usages of $C$, while issuing a request and subsequently being granted a single access to Data from the Checker TEE. The adversary first initiates the $\mathcal{F}$ TEE at state $s_{0}$, and then feeds Data to $\mathcal{F}$, causing the TEE to transit to $s_{D}$. Next, the adversary triggers the TEE to seal $s_{D}$ to secondary storage using the data sealing mechanism. Finally, it reruns $\mathcal{F}$ from $s_{D}$ multiple times, each with a separate input to obtain both $\mathcal{F}\left(\right.$ Data, $\left.X_{1}\right)$ and $\mathcal{F}\left(\right.$ Data, $\left.X_{2}\right)$.

\section{SYSTEM AND THREAT MODELS}

In this section, we describe the system model we adopt in our design, the trust assumptions in our system model, as well as the adversary model our design tackles.

\subsection{System Model}

We adopt a system model wherein the access control is regulated by a committee of nodes, namely JuRY. In addition, we simplify the notion of data capsule's free circulation by incorporating a public storage in our system model. An overview of the interplay between the parties is shown in Figure 1.

- Data Owner Owner. She wishes to encapsulate her sensitive data Data into a data capsule $C$.

- Public Storage. Without loss of generality, we equate the notion of $C$ 's free circulation to it being stored on a public storage. The generality of the system requires that its design is agnostic to the implementation of the public storage. Interactions with the storage are distilled into two main functions, namely, store (id, v) which puts the data $v$ indexed by id into the storage, and retrieve(id) which returns the data indexed by id if it has been stored. 


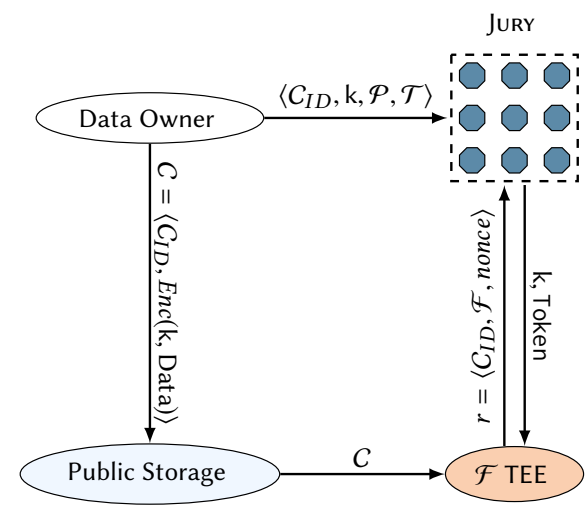

Figure 1: An overview of the interplay between the parties.

- Access Control Committee Jury. It comprises independent and mutually distrusting nodes. JURY is collectively responsible for regulating the access policies dictated by the data owners on their data capsules. Specifically, for each data capsule $C$, the committee maintains its usage transcript $\mathcal{T}$ and encryption key k. JURY serves as a unique portal via which the function $\mathcal{F}$ can obtain access to the protected data Data. Given a request to access $C$ 's Data from $\mathcal{F}$ with measurement $M_{\mathcal{F}}$, JuRY determines if $M_{\mathcal{F}} \in \mathcal{P}$ and $\operatorname{Is} \operatorname{ExpiRed}(\mathcal{T}, \mathcal{P})$ returns false, wherein $\mathcal{P}$ and $\mathcal{T}$ are $C$ 's associated access policy and usage transcript respectively. If these conditions check out, $\mathcal{F}$ gains access to the encryption key k, enabling it to consume Data in its private memory.

- $\mathcal{F}$ TEE with Attested Execution. It is required that any function $\mathcal{F}$ that wishes to access the sensitive data Data encapsulated in a data capsule $C$ must feature attested execution. Ideally, $\mathcal{F}$ runs inside a TEE (referred to as the $\mathcal{F}$ TEE) that offers protection on integrity of $\mathcal{F}$ 's execution logic, and on confidentiality of its private memory. The encryption key $\mathrm{k}$ associated with the data capsule $C$ is securely provisioned to $\mathcal{F}$ TEE's private memory via an authenticated communication channel, and never leaves the private memory.

Remarks. Access policy $\mathcal{P}$ contains a set of TEE measurements corresponding to the functions that Owner approves. This assumption offloads the task of examining the execution logic of the eligible functions to Owner. This burden is necessarily posed on Owner, for automatically determining if a function $\mathcal{F}$ 's code attempts to secretly leak sensitive data is a non-trivial (and perhaps unsolved) problem. Nodes in JuRY can then check the eligibility of a function $\mathcal{F}$ simply by verifying if its measurement $M_{\mathcal{F}}$ is contained in $\mathcal{P}$ While reducing the examination of a function's eligibility to a simple membership checking greatly simplifies the complexity of JURY, this limits the expressibility of the system. Nonetheless, we believe that this is a reasonable trade-off between security and usability. One approach to improve the system's expressibility without little downgrade of its security is to have $\mathcal{P}$ construed and the requesting functions synthesized and then verified under a well-defined specification language using program synthesis and verification techniques $[30,46]$. This challenging problem deserves a separate study which we do not attempt to include in this work.
Workflow. The data owner Owner encapsulates her sensitive data Data into a data capsule $C$, and then deposits $C$ to the Public Storage and its constituents (namely, access policy $\mathcal{P}$, encryption key $\mathrm{k}$ and usage transcript $\mathcal{T}$ ) to Jury. Any party that wishes to access $C$ 's Data using a function $\mathcal{F}$ must first provision an $\mathcal{F}$ TEE with attested execution. He then sends a request $r=\left\langle C_{I D}, \mathcal{F}\right.$, nonce $\rangle$ that specifies to seek permission from JURY to conduct a functional access of $\mathcal{F}$ on $C$ 's Data, wherein $C_{I D}$ is a unique handle of $C$, and nonce is auxiliary information that distinguishes access requests of the same $\mathcal{F}$ on the same $\mathcal{C}$. JuRY grants the $\mathcal{F}$ TEE access to $C$ 's Data by delivering $\mathrm{k}$ and a randomly generated token Token to the latter, wherein $\mathrm{k}$ is the key to decapsulate $C$, and Token is a request-specific token that prevents the double-consumption attack on $C$. Section 5.2 elaborates on the workflow that Owner, JuRY and access requester (the $\mathcal{F}$ TEE) follow to attain the desired system goals, in the form of three secure protocols.

\subsection{Assumptions and Threat Model}

Assumptions on TEEs. Our approach requires $\mathcal{F}$ to be executed in a TEE featuring attested execution. Our design assumes that the mechanism which is used to provision the TEE is implemented and manufactured properly, and that the TEE protects the execution integrity of the program loaded inside and the confidentiality of the data stored in its private memory. There exist various hardware primitives to provision TEEs, for instance, Intel SGX [33], Sanctum [15] and Keystone [27]. Although our work adopts Intel SGX, we remark that our design is compatible with other TEE instantiations. While Intel SGX admits side-channel attacks [8, 49], mitigating or hardening enclaves against these attacks $[16,37]$ is an active line of research, which is orthogonal to our work.

Assumptions on Access Control Committee JURY. Nodes in JuRY are independent and mutually distrusted. JURY is able to remain fully functional in the presence of Byzantine failures. While this assumption can be realised by having JURY implementing a Byzantine Fault Tolerent (BFT) consensus protocol such as PBFT [9], its overhead has been shown to be prohibitive $[3,17]$. To minimize such overhead, our work makes an additional assumption that each node in JuRY features a TEE. The nodes run their codebase inside a TEE with attested execution, which effectively restricts adversarial behaviours of the faulty nodes. Although the nodes may not trust each other, they trust the codebase implementing JURY's functionality, which can be vetted and formally verified. The pairwise communication between nodes is conducted via a secure and authenticated communication channel. In the absence of adversaries, these channels are synchronous, i.e., all messages are delivered within a finite delay $\Delta$ known a-priori. Let us denote by $n$ the number of nodes in JURY. A node is considered faulty if it crashes, or it cannot communicate with a quorum of $\left\lfloor\frac{n}{2}\right\rfloor+1$ nodes in a synchronous fashion. Let $f$ be the number of faulty nodes in the committee, we require $f \leq\left\lfloor\frac{n-1}{2}\right\rfloor$ in order for the committee to be fully functional.

Assumptions on $\mathcal{F}$. We assume that $\mathcal{F}$ is programed or instrumented in such a way that its execution logic proceeds in steps, each of which consumes some input $x_{i}$ to transit from state $s_{i}$ to state $s_{i+1}$. When the input to $\mathcal{F}$ is Data and a (multi-parameter) input $X=\left\{x_{1}, x_{2}, x_{3}, \ldots, x_{m}\right\}$, we can presume that $\mathcal{F}$ is initialized 
at state $s_{0}$, consuming Data to transit to state $s_{1}$, then consuming $x_{1}$ to transit to state $s_{2}$, consuming $x_{2}$ to transit to $s_{3}$, and so on until $X$ is fully consumed and $\mathcal{F}$ produces the final output $\mathcal{F}($ Data, $X)$.

Threat Model. We assume a powerful adversary that has full control over the host (or machine) on which the TEE is provisioned. Specifically, the adversary can corrupt or schedule all non-enclave processes and access their memory content. It can tamper with data persisted on the host's storage. It can also intercept, read, modify, reorder and delay messages sent from and to the enclave. Nonetheless, the adversary is computationally bounded, and cannot break standard cryptographic assumptions. Moreover, the adversary is unable to access runtime memory of an enclave or leak confidential information protected by the enclave. It also does not have any access to the processor's private keys which are utilized in attestation and data sealing.

It is worth noting that enclave computations, especially those that involve data sealing, are vulnerable to rollback attacks. Rollback attacks enable adversaries to rewind the enclave to its previous state, or even compromise the integrity of the enclave's state. Specifically, the adversary awaits the victim enclave to seal two data elements (which may be snapshots of the enclave's states) $c t_{1} \leftarrow \operatorname{Seal}\left(s_{1}\right)$ and $c t_{2} \leftarrow \operatorname{Seal}\left(s_{2}\right)$ to the host's persistent storage, with $s_{2}$ representing the enclave's more recent state. The adversary then terminates and restarts the enclave, so as to erase its volatile runtime memory. When the resuming enclave asks for its most recent sealed data $c t_{2}$, the adversary provides it with $c t_{1}$, causing the enclave to accept $s_{1}$ as its latest state and "forgetting" all computation that had happened after $s_{1}$.

As described in Section 3.4, via rollback and replay attacks on the $\mathcal{F}$ TEE, an adversary could double-consume $C$ 's Data. We incorporated measures to defense such attacks in our solution (refer to Section 5.1 for more details).

\section{OUR APPROACH}

In this section, we present TEEKAP, our platform for self-expiring data capsules. Section 5.1 provides an overview of TEEKAP, while Section 5.2 elaborates on its three main protocols. Section 5.3 discusses two design alternatives and contrasts them against TEEKAP.

\subsection{Solution Overview}

Our design focuses on addressing two key challenges. The first is to ensure the access control committee JURY, which is tasked to uphold the confidentiality of Data and the integrity of $\mathcal{T}$, remains fully functional even in the presence of Byzantine failures. TEEKAP overcome this chanllege by embracing decentralization, combining state machine replication [39] and threshold secret sharing in a novel way. The second challenge is to prevent a malicious access requester from double-consuming $C$ 's Data. TEEKAP safeguards $C$ against double-consumption attacks by binding all steps in the functional access of a function $\mathcal{F}$ on $C$ 's Data into a single session uniquely identified by a session-specific token Token generated randomly by JURY. For clarity of exposition, in the folllowing, we discuss how TEEKAP upholds the three invariants for a single data capsule $C$.

Confidentiality of Data. Provided that the encryption scheme used to produce $C$ 's ciphertext is secure, keeping k private against any unauthorised parties suffices to protect the confidentiality of Data. To this end, we adopt a threshold secret-sharing scheme $[31,40]$ to distribute $\mathrm{k}$ among nodes in JURY, decentralizing the protection of k's confidentiality. Under a $(n, t)$ secret-sharing scheme wherein $n$ is the number of nodes in JuRY and $0<t \leq n, \mathrm{k}$ is split into $n$ shares, each of which is deposited to a separate node in JuRY. Any subset of $t$ or more shares can be used to reconstruct $\mathrm{k}$, while individual share does not reveal any information about $k$. That is, the adversary must compromise at least $t$ nodes and obtain their shares in order to extract $k$. The secret-sharing scheme also enhances robustness of the system against independent node failures, for $k$ is only rendered unattainable when more than $n-t$ shares are lost.

Integrity of $\mathcal{T}$. Given that all accesses to $C$ are recorded in its associated usage transcript $\mathcal{T}$, which is then used to determine the expiry status of $C$, thus, we need to safeguard $\mathcal{T}$ from tempers or falsifications. TEEKAP leverages replication techniques to ensure the integrity of $\mathcal{T}$. That is, $\mathcal{T}$ is replicated across independent nodes. These nodes run a distributed consensus protocol to ensure that their local copies of $\mathcal{T}$ converge even in the presence of individual node failures or adversaries.

Examining and subsequently granting an access request can be deemed as a distributed read-then-write transaction to $\mathcal{T}$, for it is replicated across independent nodes. When there are concurrent access requests to $C$, each of which is valid and eligible should it be considered independently, granting one request may render the other request ineligible. As such, concurrency control techniques, such as two-phase locking [24], are required in the processing of access requests.

Defense Against Double-Consumption of Data. Ensuring the integrity and validity of the usage transcript $\mathcal{T}$ alone is not sufficient to enforce $C$ 's expiry, for the adversary could launch a rollback and replay attack on the $\mathcal{F}$ TEE to double-consume Data locally, as described in Section 3.4. To mitigate such attacks, TEEKAP binds all the steps in the evaluation of $\mathcal{F}($ Data, $X)$ into a single session, which is uniquely identified by a session-specific token Token generated randomly by JURY. In particular, the execution logic of the $\mathcal{F}$ TEE proceeds in steps, each of which consumes an input $x_{i}$ and a token token ${ }_{i}$ to transit $\mathcal{F}$ 's state from $s_{i}$ to $s_{i+1}$. At $\mathcal{F}$ 's initial state $s_{0}$, the $\mathcal{F}$ TEE receives Token from JURY as token , consuming $^{2}$ Data to transit to state $s_{1}$. Thereafter, $\mathcal{F}$ transits from $s_{i}$ to $s_{i+1}$ (i.e., its execution proceeds) if and only if token $_{i}=$ token $_{i-1}$. The $\mathcal{F}$ TEE uses Token in place of token $i$ for all $i=(1,2, \ldots, n)$ in its computation, thereby binding all steps in the evaluation of $\mathcal{F}($ Data, $X)$ into a single session. It is worth mentioning that JuRY delivers the Token to the $\mathcal{F}$ TEE via a secure and authenticated communication channel, and the $\mathcal{F}$ TEE maintains the Token exclusively in its private memory. That is, Token is not sealed to a secondary storage even if the $\mathcal{F}$ TEE persists some of its data and/or state via the data sealing mechanism, which prevents the adversary from ever obtaining Token.

Remarks. Decentralization in our context goes beyond replication or redundancy. Depositing the secret's shares to independent nodes offers better protection for the confidentiality of the secret, this is so because compromising a set of independent nodes in a coordinate manner is clearly more arduous than doing so on a single node. 


\subsection{Protocol Details}

We now elaborate on the workflow of TEEKAP, in the form of three main protocols, namely, ENCAPSULATE for a data owner who wishes to encapsulate her sensitive data into a data capsule, REQUESTACCESS for an access requester who wishes to access the sensitive data using function $\mathcal{F}$, and ProcessRequest for the access control committee JURY to process the received access requests. JURY consists of $n$ independent nodes $\left\langle N_{1}, N_{2}, \ldots, N_{n}\right\rangle$, each of which runs ProcessRequest inside an enclave.

ENCAPSULATE. The data owner Owner follows the following steps to encapsulate Data into a data capsule $C$ :

(E1) Owner picks an encryption key k uniformly at random.

(E2) Owner encrypts Data with k using a symmetric encryption scheme [26], obtaining $C=\left\langle C_{I D}\right.$, Enc(k, Data) $\rangle$, wherein $\operatorname{Enc}(\mathrm{k}$, Data $)$ is Data's ciphertext, $C_{I D}=H(\mathrm{Enc}(\mathrm{k}, \mathrm{Data}))$ is a handle for $C$, and $H()$ is a cryptographic hash function [26].

(E3) Owner uses a $(n, t)$ threshold secret-sharing scheme to split $\mathrm{k}$ into $n$ shares $\left(\mathrm{k}_{1}, \mathrm{k}_{2}, \ldots, \mathrm{k}_{n}\right)$ such that any subset of $t=$ $\left\lfloor\frac{n}{2}\right\rfloor+1$ or more shares can be used to reconstruct k.

(E4) Owner creates the access policy $\mathcal{P}$ and the initial usage transcript $\mathcal{T}$ for data capsule $C . \mathcal{P}$ contains a list of enclave measurements representing functions that can access Data and the threshold values for expiry condtions. Here, $\mathcal{P}$ and $\mathcal{T}$ are uniquely identifiable by $C$ 's handle $C_{I D}$.

(E5) Owner deposits $\left\langle C_{I D}, \mathrm{k}_{i}, \mathcal{P}, \mathcal{T}\right\rangle$ to node $N_{i}$ in JuRY $(1 \leq i \leq$ $n$ ) via a secure and authenticated channel.

(E6) Owner stores $C$ to the Public Storage, indexing it with $C_{I D}$. Remarks. Our platform also allows Owner to modify $\mathcal{P}$. Such modification requires a consensus protocol run among JuRY nodes so that the modification to $\mathcal{P}$ is reflected at all non-faulty nodes. The interaction between nodes in JURY in this consensus run is similar to the one described in the ProcessRequest protocol below.

REQUESTACCESS. An access requester $\mathrm{R}$, who wishes to access data capsule $C$ using a function $\mathcal{F}$, follows the following steps:

(R1) R instantiates the enclave $E_{\mathcal{F}}$ that hosts $\mathcal{F}^{\prime}$ 's execution. During enclave initialization, a public-private key pair $\left(\mathrm{pk}_{\mathcal{F}}, \mathrm{sk}_{\mathcal{F}}\right)$ is generated uniformly at random, which can be used by a remote party to establish a secure and authenticated communication channel with $E_{\mathcal{F}}$.

(R2) R obtains $E_{\mathcal{F}}$ 's remote attestation report $\pi_{\mathcal{F}}=\left\langle M_{\mathcal{F}}, \mathrm{pk}_{\mathcal{F}}\right\rangle_{\sigma_{T E E}}$ from the trusted processor. $M_{\mathcal{F}}$ is the enclave's measurement, and $\sigma_{T E E}$ is a group signature ${ }^{5}$ signed by the processor's attestation key.

(R3) $\mathrm{R}$ requests Intel Attestation Service for SGX (IAS) [13] to verify $\pi_{\mathcal{F}}$, retrieving $\operatorname{Cert}_{\mathcal{F}}=\left\langle\pi_{\mathcal{F}} \text {, valid }\right\rangle_{\sigma_{I A S}}$ as a response, wherein $\sigma_{I A S}$ is a publicly verifiable signature of IAS. That is, $\operatorname{Cert}_{\mathcal{F}}$ is a publicly verifiable certificate that proves the correct instantiation of $E_{\mathcal{F}}$.

(R4) R sends a request of the form $r=\left\langle C_{I D}\right.$, Cert $_{\mathcal{F}}$, nonce $\rangle$ to JuRY (e.g., to the current leader of JuRY). JuRY will use the

\footnotetext{
${ }^{5}$ We use the Intel EPID-based Intel Attestation Service for SGX (IAS) to explain our protocols in this section. Note that other ECDSA-based third-party attestation services can fit into our protocols seamlessly [12].
}

ProcessRequest protocol described below to process request $r$. Here, nonce is a 64-bit random number sampled uniformly, and is unique for each request. This nonce is used to distinguish requests for functional access of the same function $\mathcal{F}$ on the same data capsule $C$.

(R5) $E_{\mathcal{F}}$ awaits for responses from a quorum of $\left\lfloor\frac{n}{2}\right\rfloor+1$ nodes in $\mathrm{JURY}^{6}$. Each such response is either $\perp$ (access request denied) or of the form $\left\langle\mathrm{k}_{i}\right.$, Token $\rangle$, wherein $\mathrm{k}_{i}$ is a key share kept by a node $N_{i}$ in JuRY and Token is a request-specific token generated in step (P2) of the ProcessRequest protocol described below. If this access request is not denied, once $E_{\mathcal{F}}$ has obtained sufficient number of responses with matching Token, it reconstructs the decryption key $\mathrm{k}$, and uses $\mathrm{k}$ and Token to evaluate $\mathcal{F}($ Data, $X)$ for some $X$ provided by the access requester $\mathrm{R}$.

PRocessRequest. Leveraging the Raft [38] consensus protocol, it ensures that local copies of $\mathcal{T}$ in JURY nodes converge. Following Raft, one node in JURY is elected as a leader ${ }^{7}$, while others are followers. For clarity, let us denote the leader as $L$. Upon receiving a request $r=\left\langle C_{I D}\right.$, Cert $t_{\mathcal{F}}$, nonce $\rangle$ from an access requester, $L$ coordinates the processing of the request as follows:

(P1) $L$ first verifies if $\operatorname{Cert}_{\mathcal{F}}=\left\langle\pi_{\mathcal{F}} \text {, valid }\right\rangle_{\sigma_{I A S}}$ is authenticated by the IAS by examining $\sigma_{I A S}$. Next, it checks if $M_{\mathcal{F}} \in \mathcal{P}$ and $\operatorname{IsExPIRED}(\mathcal{T}, \mathcal{P})$ returns false. If all these conditions check out, $L$ proceeds to the next step. Otherwise, it responds the access requester with $\perp$, indicating denial of access request.

(P2) $L$ samples uniformly a 64-bit random number as a token Token and uniquely binds it to the request $r$. $L$ logs an entry $\langle r$, Token $\rangle$ in its cache, before broadcasting it to all followers.

(P3) Upon receiving $\langle r$, Token $\rangle$, a follower $N_{i}$ performs the same checks on $r$ that $L$ have done in step (P1). If all the conditions check out, $N_{i}$ responds to $L$ with an acknowledgement $\left\langle a c k_{i}, r\right\rangle$, and logs $\langle r$, Token $\rangle$ in its cache. Next, it establishes a secure and authenticated communication channel to the enclave $E_{\mathcal{F}}$ (using $\mathrm{pk}_{\mathcal{F}}$ found in $\operatorname{Cert}_{\mathcal{F}}$ ), and delivers $\left\langle\mathrm{k}_{i}\right.$, Token $\rangle$ to the latter, where $\mathrm{k}_{i}$ is the key share it keeps.

(P4) Once $L$ has confirmed that $r$ has been replicated on a majority of the nodes in JURY, it adds $\langle r$, Token $\rangle$ to $\mathcal{T}$, signalling that $E_{\mathcal{F}}$ has been granted an access to $C$ 's Data under the requestspecific token Token. Subsequently, it also sends the key share it keeps together with Token to $E_{\mathcal{F}}$ via a secure and authenticated channel. By this time, $L$ has committed the update of $\mathcal{T}$, reflecting an access of $\mathcal{F}$ on $C$ 's Data under the request-specific token Token. $\langle r$, Token $\rangle$ may be now discarded from $L$ 's cache.

(P5) If this update to $\mathcal{T}$ renders $C$ expired, i.e., $\operatorname{IsExPIRED}(\mathcal{T}, \mathcal{P})$ returns true, $L$ discards the key share it keeps, and announces the commit in the next message it exchanges with followers.

(P6) Once a follower $N_{i}$ sees the commit in step (P4), it adds $\langle r$, Token $\rangle$ to $\mathcal{T}^{8}$. If this leads to the expiration of $C, N_{i}$ discards the share $\mathrm{k}_{i}$ associated with $C$.

\footnotetext{
${ }^{6}$ Each Jury node establishes a secure and authenticated channel to communicate the key share to the enclave $E_{\mathcal{F}}$ using its public key $\mathrm{pk}_{\mathcal{F}}$ embedded in Cert $\mathrm{F}_{\mathcal{F}}$.

${ }^{7}$ When the leader fails, it is replaced by a new one via the leader election protocol.

${ }^{8}\langle r$, Token $\rangle$ may now be discarded from $N_{i}$ 's cache.
} 
Remarks. In steps (P3) and (P4), nodes in JuRY separately deliver key shares to the enclave $E_{\mathcal{F}}$. One may think of an alternative wherein a follower $N_{i}$ piggybacks its share $\mathrm{k}_{i}$ on the acknowledgement it responds to the leader $L$ in step (P3), then $L$ reconstructs the key once it has received a quorum of acknowledgements and subsequently delivers the key securely to the enclave $E_{\mathcal{F}}$ in step (P4). While this approach avoids communication between the followers and the enclave $E_{\mathcal{F}}$, it places heavy stress on the communication channel between $L$ and the enclave $E_{\mathcal{F}}$. In particular, if the adversary manages to eclipse their communication channel, it may forward the key delivery elsewhere and thereby make the access granting to the enclave $E_{\mathcal{F}}$ to fail, even though the $\mathcal{T}$ associated with the data capsule in question records $\mathcal{F}$ 's functional access. This inconsistency is problematic, for it may also deny an otherwise valid and eligible request. Concretely, let us consider two requests $r_{1}$ and $r_{2}$ submitted when the availability status of the data capsule $C$ allows only one more access. If $r_{1}$ is recorded in the $\mathcal{T}$ associated with $C$ as being granted, but the key $k$ is not delivered to $r_{1}$ 's targeted enclave, consequently, $r_{1}$ actually never accesses $C$, while $r_{2}$ is still denied. It clearly opens up wider denial-of-service attack vector for the adversary.

\subsection{Discussion: Splitting JuRY}

In TEEKAP, the threshold $t$ of the secret-sharing scheme is set in accordance with the quorum size $q$ of the consensus protocol in use (for Raft, $q=\left\lfloor\frac{n}{2}\right\rfloor+1$ ), with $q \leq t \leq n$. That is, the choice of the committee size becomes delicate. A large committee size makes it more difficult for the adversary to extract the secret keys (as the adversary has to compromise more nodes in order to obtain the required shares), but may lessen the JuRY's throughput due to higher communication overhead [3, 17]. In contrast, a small commitee size enables JuRY to afford high throughput, but lowers the robustness of the system against attacks that aim to illicitly extract the encryption keys.

Therefore, to attain flexibility in configuring system and security parameters, alternative designs are to split JURY into two committees; one safeguards the usage transcripts $\mathcal{T}_{\mathrm{s}}$, and the other one the encryption keys ks.

Separate Committees. One can split JuRY into two separate committees, namely JURY $\mathrm{JU}_{\mathrm{k}}$ and JURY $\mathcal{T}$. JURY $\mathrm{JU}_{\mathrm{k}}$ is responsible for safeguarding $k$, whereas JURY $\mathcal{T}$ is tasked to keep track of $\mathcal{T}$. This approach necessarily requires coordination between operations of $\mathrm{JURY}_{k}$ and $\operatorname{JURY}_{\mathcal{T}}$. It is needless to say that nodes in $\mathrm{JURY}_{k}$ do not have permission to modify $\mathcal{T}_{\mathrm{s}}$, and nodes in $\mathrm{JURY}_{\mathcal{T}}$ do not have any knowledge of ks or their shares.

Having two separate committees allows greater flexibility in configuring system and security parameters. For instance, JURY ${ }_{k}$ may comprise a large number of commodity nodes, while JURY $\mathcal{T}$ can be configured to consist of only a small number of nodes with defencein-depth mechanisms $[22,42]$ in place to better safeguard them against adversarial attempts. Nonetheless, the separation of JURY and $\operatorname{JURY}_{\mathcal{T}}$ presents challenges in coordinating the interactions between the access requester $\mathrm{R}$ and the two committees.

Granting $\mathcal{F}$ access to $C$ 's Data entails updating $\mathcal{T}$ and delivering shares of $\mathrm{k}$ to the enclave $E_{\mathcal{F}}$. This is essentially a distributed and atomic transaction [41]. TEEKAP would need to involve an atomic commitment protocol, such as two-phase commit protocol (2PC) [4], to handle such transactions. Unfortunately, this will add significant complications to the system. First, since JURY $\mathrm{J}_{\mathrm{k}}$ and $\mathrm{JURY}_{\mathcal{T}}$ are collections of nodes, each action in an atomic commitment protocol entails a complete run of a consensus protocol in one committee. Second, committee-to-committee communication poses heavy stress on the network when the committees are large, which likely leads to message drops, which in turn exacerbates the intra-committee's consensus protocol runs.

Overlapping Committees. Yet another design choice is to have the access committee comprising a large number of nodes, but not all of them are responsible for handling the usage transcript $\mathcal{T}$ s. In particular, given a data capsule $C$ with encryption key k, each node in the committee keeps a single share of $k$, but only a select few nodes are tasked to maintain replicated copies of its usage transcript $\mathcal{T}$. This is equivalent to a design choice that engages two committees, each of which defends against one invariant, but one committee is a strict subset of the other. We abuse the notation and denote these two committees by JURY ${ }_{\mathrm{k}}^{\prime}$ and $\mathrm{JURY}_{\mathcal{T}}^{\prime}$, then $\mathrm{JURY}_{\mathcal{T}}^{\prime} \subset$ $\mathrm{JURY}_{\mathrm{k}}^{\prime}$. More generally, let us consider a setting in which $\mathrm{JURY}_{\mathcal{T}}^{\prime}$ may not be a subset of JURY $\mathrm{k}_{\mathrm{k}}^{\prime}$, but there exists a non-empty subset of nodes that belong to both JURY $\mathcal{T}_{\mathcal{T}}^{\prime}$ and $\operatorname{JURY}_{\mathrm{k}}^{\prime}\left(\right.$ i.e., $\operatorname{JURY}_{\mathcal{T}}^{\prime} \cap \operatorname{JURY}_{\mathrm{k}}^{\prime} \neq \emptyset$ ).

Similar to the design choice that uses two separate committees, this setting allows flexibility in configuring system and security parameters. That is, one can configure different committee sizes for $\mathrm{JURY}_{\mathrm{k}}^{\prime}$ and $\mathrm{JURY}_{\mathcal{T}}^{\prime}$; larger JURY ${ }_{\mathrm{k}}^{\prime}$ makes the committee more robust in safeguarding the confidentiality of encryption keys, whereas smaller $\mathrm{JURY}_{\mathcal{T}}^{\prime}$ yields higher throughput in processing the repli-

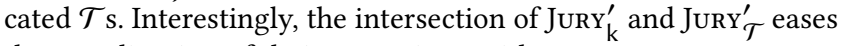
the coordination of their operations with respect to an access request. In fact, we believe that it is possible to extend the protocol design constructed for JURY to support the interactions between $\mathrm{JURY}_{\mathrm{k}}^{\prime}$ and $\mathrm{JURY}_{\mathcal{T}}^{\prime}$, albeit more protocol parameters and convoluted protocol details. However, the aforementioned flexibility in system configurations and ease of coordination come at a price of complications in defining the threat model governing $\operatorname{JURY}_{\mathrm{k}}^{\prime}$ and $\mathrm{JURY}_{\mathcal{T}}^{\prime}$, and reasoning about the security of the system with respect to such a threat model. This is so because faulty nodes belonging to the intersection of JURY ${ }_{\mathrm{k}}^{\prime}$ and $\mathrm{JURY}_{\mathcal{T}}^{\prime}$ may entail different security implications in comparison with those that do not belong to the intersection. We leave this interesting exploration to future work.

Remarks. In view of the challenges and complications in the two design alternatives described above, TEEKAP employs a single committee, which alleviates the need of coordinating the interplay between updating $\mathcal{T}$ and delivering $\mathrm{k}$ to the enclave $E_{\mathcal{F}}$, naturally ensuring atomicity of granting access request. In addition, this design choice requires JURY to undertake only one consensus protocol run per access request, which is more efficient and incurs lower latency than the $2 \mathrm{PC}$-based coordination between $\mathrm{JURY}_{\mathrm{k}}$ and $\mathrm{JURY}_{\mathcal{T}}$ described in the design choice that involves two separate, non-overlapping committees.

Compared with the above two design alternatives, TEEKAP argurably puts more constraints on configuring system and security parameters. Nonetheless, we believe that this is a reasonable tradeoff between security and performance. 


\section{SECURITY ARGUMENTS}

In this section, we first reason about the protections and guarantees offered by TEEs, and how they affect the security of TEEKAP. We then provide security arguments to justify how TEEKAP attains the system goals presented in Section 3.2. Our security arguments build on that of the secret-sharing scheme [40] and that of the Raft consensus protocol [38].

\subsection{TEE Protections and Guarantees}

TEEKAP assumes that the adopted TEE guarantees both integrity of code execution inside its private memory, and confidentiality for data stored there.

Execution Integrity Protection. TEEKAP relies on the execution integrity protection of enclave $E_{\mathcal{F}}$ to prevent the adversary from deviating the authorised function $\mathcal{F}$ from its intended execution logic, or having $\mathcal{F}$ explicitly misuse or persist Data or the key materials outside of the enclave memory. Likewise, enclaves are also assumed to safeguard the execution integrity of nodes in JURY, thereby allowing ProcessRequest to build on Raft [38] and enjoy its safety guarantee. The assumption on integrity protection has been generalised under a Sealed-Glass Proof model [44], and it is hardly challenged by attacks against TEEs.

Data Confidentiality Protection. TEEKAP also leverages the confidentiality protection of enclave $E_{\mathcal{F}}$ to keep the request-specific token Token, the encryption key $\mathrm{k}$ and the protected data Data confidential in enclave $E_{\mathcal{F}}$ 's private memory. Nonetheless, it is worth acknowledging that TEE's confidentiality protection remains a strong and somewhat wishful assumption in the presence of side-channel attacks against TEEs [8, 49]. Although our work does not attempt to handle these attacks, there exists an active line of research whose primary purpose is to harden enclave execution against such a threat $[16,37]$. These measures can be incorporated into TEEKAP to enhance its confidentiality protection.

Notwithstanding the above argument, it is useful to discuss the consequences arised when an adversary successfully compromises TEE's confidentiality protection, except for a handful of its important cryptographic primitives: attestation, key generation, data sealing, and signature (i.e., the Sealed-Glass Proof model [44]). One on hand, such an adversary cannot obtain key shares from nodes in JURY, for they do not need to maintain the shares in their enclave memory. Instead, they can seal the shares to a persistent storage using the CPU-generated key uniquely bound to enclave measurement. On the other hand, should the adversary successfully compromise confidentiality protection of $E_{\mathcal{F}}$, the adversary can extract Token, $k$, and Data. Extracting Token alone enables the adversary to double-consume Data, while extracting either $k$ or Data would give the adversary unlimited access to the protected data. Fortunately, the use of Token in the execution of $E_{\mathcal{F}}$ does not depend on either Data or the supplementary input $X$ provided by the access requester, which implies the adversary would have a harder time in leaking Token using side-channel attacks. In the worst case where $E_{\mathcal{F}}$ is compromised and the key is leaked, this would allow the adversary unrestricted access to Data in question. However, this does not put other data capsules in jeopardy, for they use different encryption keys.

\subsection{Upholding Access Control Criteria and Enforcing Data Expiry}

We first show that the access control committee JuRY enforces the access control criteria imposed on a data capsule $C$. Based on remote attestation mechanisms [1] and the fact that $\operatorname{Cert}_{\mathcal{F}}$ is publicly verifiable, each node in JURY can independently determines if the enclave $E_{\mathcal{F}}$ is correctly instantiated, if $M_{\mathcal{F}} \in \mathcal{P}$, and if $\operatorname{Is} \operatorname{ExpIRED}(\mathcal{T}, \mathcal{P})$ returns false. The ProcessReguest protocol follows Raft [38] to ensure that the nodes in JURY maintain a consistent view of $C$ 's usage transcript $\mathcal{T}$, and that $\mathcal{F}$ could obtain $\mathrm{k}$ only when the nodes have reached a consensus.

It is worth mentioning that the use of Token effectively binds all steps in the computation of $E_{\mathcal{F}}$ into a single session, which is tied to a specific request $r$ approved by JuRY. As long as the adversary is not able to obtain Token, it cannot meaningfully rollback $E_{\mathcal{F}}$ 's execution, thereby not being able to double-consume C's Data.

Next, we discuss how ProcessRequest effectuates $C$ 's expiry, rendering Data permanently unattainable. Recall that the expiry status of $C$ is determined based on its usage transcript $\mathcal{T}$. In steps (P5) and (P6) of ProcessRequest, nodes in Jury discard their shares of $k$ as soon as there is an update to $\mathcal{T}$ that leads to expiration of $C$. ProcessRequest follows Raft [38] to ensure that local copies of $\mathcal{T}$ at each node in JuRY converge, thereby having the key shares collectively discarded when $\operatorname{IsEx} \operatorname{PiRED}(\mathcal{T}, \mathcal{P})$ becomes true. This disposes k, effectively rendering Data unattainable thereafter.

Enforcing expiry requires those functions that retrieved $\mathrm{k}$ prior to $C$ 's expiry do not intentionally leak or persist $k$ or Data outside of enclave memory. This assurance is covered under the eligibility of $\mathcal{F}$, which can be attested using romote attestation mechanisms [1]. That is, the data owner has examined $\mathcal{F}$ 's code and ascertained that $\mathcal{F}$ does not have the above malicious intentions.

\subsection{Rollback Attacks on JURY}

As presented earlier, TEEs are susceptible to rollback attacks [7, 32] wherein an adversary (e.g., the malicious OS) provides the resuming enclave with properly sealed but stale data. The use of requestspecific token Token prevents such an adversary from perpetrating rollback attacks on the enclave $E_{\mathcal{F}}$. Nonetheless, the adversary may also attempt this attack on TEEs in JURY nodes so as to unwind the usage transcript $\mathcal{T}$ associated with an already expired data capsule, making it available again. Alternatively, the adversary whose eligibility has been revoked by the data owner could try to undo such a revocation by triggering the nodes in JURY to work with a stale access policy. Fortunately, these attacks on JURY are not possible under our threat model.

In order to carry out a rollback attack on a node's TEE, the adversary has to first crash its TEE, and then restore the TEE with a stale $\mathcal{T}$. Since our threat model restricts that the number of faulty nodes in the committee $f \leq\left\lfloor\frac{n-1}{2}\right\rfloor$, the adversary cannot rollback a majority of nodes in JURY. Recall that in Raft [38], records in the replicated log flow in one direction from the leader to followers. Thus, as long as a rollbacked node is not elected as leader in TEEKAP, our ProcessRequest protocol is safe. This is clearly the case, for a candidate must obtain a quorum of votes from a majority of JURY nodes to become the leader, which only happens when its $\mathcal{T}$ is at least as up-to-date as those of the voters. 
Table 1: Network latency (ms) between Jury nodes on Azure.

\begin{tabular}{|l|c|c|}
\hline Datacenter & East US & Canada Central \\
\hline \hline East US & 0.8 & 12.7 \\
\hline Canada Central & 12.7 & 1.2 \\
\hline
\end{tabular}

\section{IMPLEMENTATION}

In this section, we describe the implementation details of our prototype TEEKAP ${ }^{9}$ using Intel SGX. We port NuRaft ${ }^{10}$, a C ++ implementation of Raft, to SGX using the Open Enclave SDK ${ }^{11}$, and refer to this implementation of Raft as Raft-SGX. For threshold secret sharing, we use Shamir's secret sharing scheme. In addition, we utilize Mbed TLS ${ }^{12}$ for secure communication and cryptographic operations, such as generation of random keys, encryption and decryption. Our Public Storage is implemented using RocksDB ${ }^{13}$, a key-value store for fast storage environments.

In our prototype, an eligible function $\mathcal{F}$ is represented by the measurement of its hosting enclave, i.e., the MRENCLAVE value of an initialized enclave. That is, the access policy that admits $\mathcal{F}$ 's functional access uses the value of MRENCLAVE as $\mathcal{F}$ 's identity.

Utilizing Raft-SGX, each JURY node implements the functionality required by the protocols Encapsulate and ProcessRequest. It also runs a TCP server, implemented using Boost.Asio ${ }^{14}$, to receive requests from data owner Owner and access requester R. Note that only the leader node processes the requests; follower nodes simply forward the received requests to the leader node. Besides, JURY uses the ECDSA-based Intel DCAP [14] attestation service for remote attestation of the requesting enclave $E_{\mathcal{F}}$ of access requester $\mathrm{R}$.

\section{EVALUATION}

This section reports our experimental study of TEEKAP, focusing on throughput and latency of ProcessRequest and Encapsulate. We conduct all experiments on Microsoft Azure using SGX-enabled virtual machines (VMs) backed by the Intel Xeon E-2288G processor, i.e., DCsv2-series Confidential Computing ${ }^{15}$ VMs. We chose size "Standard_DC4s_v2" for all VMs, each configured with 4 vCPUs, 112 MiB EPC memory, $16 \mathrm{GiB}$ RAM, and $30 \mathrm{GiB}$ SSD. At the time of our experiments, Azure only provides this series of VMs in its "East US" and "Canada Central" datacenters. We report the communication latency between nodes in these two datacenters in Table 1 .

In all experiments, we run each JuRY node in a separate VM. We run Owner, access requester $\mathrm{R}$ and Public Storage on our local machines, each equipped with the Intel Core i7-8700 processor (with Intel SGX), 16 GiB RAM and $1 \mathrm{TiB}$ SSD. All VMs/machines run Ubuntu 20.04 (amd64). For Raft, one critical parameter to be set is the election timeout value, which is influenced by the communication latency between JuRY nodes. In our experiments, we set the timeout range to be $[200-400](m s)$ for Raft-SGX.

We conduct experiments in two different settings. In the first setting, we deploy all JuRY nodes within a single datacenter, namely

\footnotetext{
${ }^{9}$ Our prototype is publicly available at https://github.com/TEELinker/TEEKAP

${ }^{10}$ https://github.com/eBay/NuRaft

$11 \mathrm{https} / / /$ openenclave.io/sdk

12 https://www.trustedfirmware.org/projects/mbed-tls

13 https://rocksdb.org

${ }^{14}$ https://www.boost.org

${ }^{15}$ https://azure.microsoft.com/en-us/solutions/confidential-compute
}

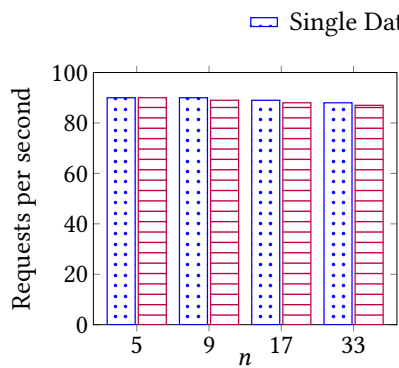

(a) ProcessRequest

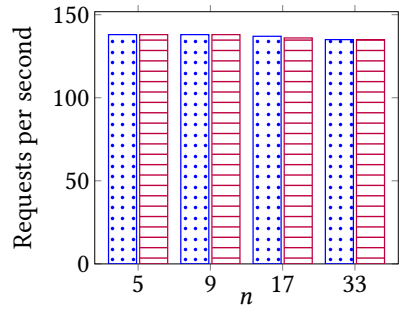

(b) ENCAPSUlate
Figure 2: Throughput of ProcessRequest and Encapsulate with respect to different Jury sizes $(n)$ on Azure.

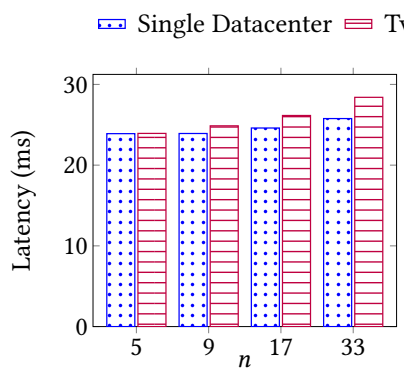

Figure 3: Raft-SGX consensus time with respect to different JURY sizes $(n)$ on Azure.

the "East US" datacenter. In the second setting, we deploy the JuRY nodes evenly across the two datacenters, with the initial leader in the "East US" datacenter. We focus on normal operation, and do not report performance of the system when the leader crashes or during leader election. Unless otherwise stated, the results presented in this section are averaged over 10 independent runs.

Figure 2 shows throughput of ProcessRequest and ENCAPSUlate with respect to different JuRY sizes $(n)$. We observe that the respective throughput remains nearly unchanged as JuRY's size grows. In addition, the respective throughput recorded on the two-datacenter setting is nearly identical with that of the one-datacenter setting. We remark that JURY's throughput is compute-bound, especially on the leader node. That is, JuRY's throughput grows with the computing power of its constituting nodes, up to a certain threshold.

To investigate the above counter-intuitive observations, we measure the consensus time (which is the main factor affecting throughtput) incurred by JuRY of different sizes $(n)$. We report the results in Figure 3. In the single-datacenter setting, the consensus time ranges from $23.90 \mathrm{~ms}(n=5)$ to $25.77 \mathrm{~ms}(n=33)$, while it ranges from $23.93 \mathrm{~ms}(n=5)$ to $28.41 \mathrm{~ms}(n=33)$ in the two-datacenter setting. We attribute these counter-intuitive observations to the stable and fast network connections between JURY nodes.

Figure 4 presents the latency incurred by ProcessRequest and ENCAPSULATE with respect to different JuRY sizes (n). In both experimental settings, the respetive latency is almost unaffected by JURy's size. In the single-datacenter setting, the latency ranges from $1033.5 \mathrm{~ms}(n=5)$ to $1045.1 \mathrm{~ms}(n=33)$, while it ranges from 1034.6 $\mathrm{ms}(n=5)$ to $1051.2 \mathrm{~ms}(n=33)$ in the two-datacenter setting. 


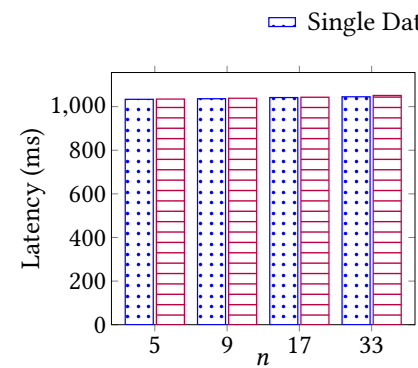

(a) ProcessRequest

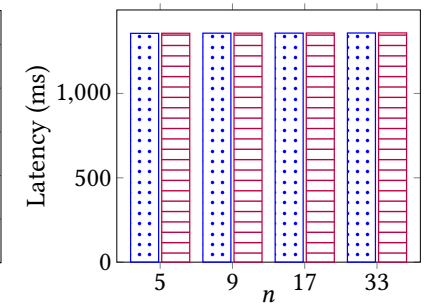

(b) Encapsulate
Figure 4: Latency of ProcessRequest and Encapsulate with respect to different Jury sizes $(n)$ on Azure.

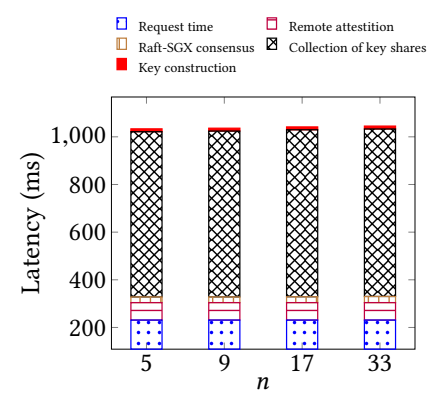

(a) ProcessRequest

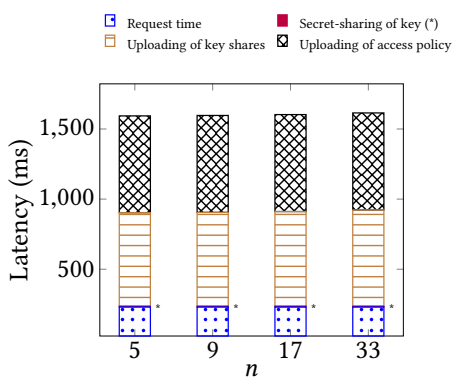

(b) Encapsulate
Figure 5: Breakdown of the latency of ProcessRequest and EnCapsulate with respect to different Jury sizes $(n)$ on Azure.

Figure 5 reports the breakdown of the respective latency incurred by ProcessRequest and Encapsulate. For ProcessRequest, the latency corresponding to JURy (i.e., "Remote attestation" and "RaftSGX consensus") is quite stable and short. In particular, "Remote attestation" takes about $73.3 \mathrm{~ms}$, whereas consensus time ranges from $23.9 \mathrm{~ms}(n=5)$ to $25.8 \mathrm{~ms}(n=33)$. For ENCAPSUlATE, most of the latency is attributed to network communication. It should be mentioned that these tasks are executed in parallel using multiple threads where possible, rather than sequentially.

In summary, TEEKAP's performance and security scale well in stable and fast network environments like public clouds.

\section{RELATED WORK}

Self-Expiring Data Object. Previous works [23, 48] have explored self-destructing/expiring data objects, wherein the access policies and expiry conditions are mainly based on timing control. TEEKAP, however, allows data owners to define generic expiry conditions.

Leveraging distributed hash table (DHT), Geambasu et al. built a system called Vanish [23]. Specifically, sensitive data is encrypted, and the encryption key is split into shares and then distributed to the DHT. Vanish ensures that the key shares will be disappered from the DHT after the set expiry time, rendering the protected data unattainable thereafter. Xiong et al. proposed FullPP [48], combining timed-release encryption [10] and DHT. The sensitive data is first encrypted into a data-ciphertext. The key used to decrypt the said ciphertext is then further encrypted into a key-ciphertex which is combined with data-ciphertext to create ciphertext shares that are distributed to the DHT. The protocol ensures that both the key and the ciphertext are destructed after the set expiry time.

Besides, previous works cannot ensure secure functional access to the protected data. TEEKAP, however, ensures that only authorized code can see the protected raw data. Neither users nor the untrusted host OS or cloud infrastructure can ever see the raw data.

TEE-enhanced Smart Contract (TEE-SC). TEE-SC $[6,11]$ is another research line that is related to our work. In their threat models, they do not actively guard against rollback and replay attacks on the TEE; in such attacks, the adversary seals the desired enclave state so that he can subsequently resume enclave execution from this state and then provide different inputs.

TEE-SC aims to ensure confidentiality of smart contract execution, while our goal is to enforce access control mechanism. Given the generality of TEE-SC, we could attempt to build a solution upon it for our problem, with the main idea of using smart contract to maintain the integrity of usage transcript. However, to uphold the confidentiality of the encryption keys, a separate Provision Service (for PDO [6]) or Key Management Committee (for Ekiden [11]) is required; this is more complex and expensive than TEEKAP.

Self-Emerging Data Objects. Standing in contrast to self-expiring data objects are self-emerging data objects. In a nutshell, sensitive data is encrypted and thus remains unreadable until a predefined time in the future. Ning et al. [34] proposed a construction that leverages threshold secret sharing and smart contracts. Specifically, the secret is divided into shares and entrusted to a group of incentivized participants, with rewards for punctual release of the shares contractualized and enforced by the autonomous smart contract. Li et al. [28] developed a self-emerging data storage wherein keys of encrypted data are given to nodes in a DHT, and revealed only after a predetermined time by routing the shares in a deterministic way. Dragchute [47], on the other hand, employs time-consuming computations to incorporate timing control into the protocol.

\section{CONCLUSION}

We proposed and formulated the problem of self-expiring data encapsulation that supports functional access and generic userdefined expiry conditions in an autonomous manner. We identified two important pieces of information necessitated in implementing self-expiring data capsules, and the technical challenges in guarding them against attacks. We presented our solution, TEEKAP, that addresses the technical challenges by integrateing TEE, consensus protocol, and threshold secret sharing in a novel way. We built a prototype of TEEKAP, conducted experiments in realistic deployment settings, and empirically showed the efficiency of our solution.

\section{ACKNOWLEDGMENTS}

This research is supported by the National Research Foundation, Singapore under its Strategic Capability Research Centres Funding Initiative. Any opinions, findings and conclusions or recommendations expressed in this material are those of the author(s) and do not reflect the views of National Research Foundation, Singapore. We thank the anonymous reviewers for their helpful feedback and insightful suggestions. 


\section{REFERENCES}

[1] Ittai Anati, Shay Gueron, Simon Johnson, and Vincent Scarlata. 2013. Innovative Technology for CPU Based Attestation and Sealing. In Proceedings of the 2nd International Workshop on Hardware and Architectural Support for Security and Privacy (HASP '13).

[2] Jason Bay, Joel Kek, Alvin Tan, Chai Sheng Hau, Yongquan Lai, Janice Tan, and Quy Tang Anh. 2020. BlueTrace White Paper. https://bluetrace.io/static/ bluetrace_whitepaper-938063656596c104632def383eb33b3c.pdf. Last accessed 2021-09-15.

[3] Johannes Behl, Tobias Distler, and Rüdiger Kapitza. 2017. Hybrids on Steroids SGX-Based High Performance BFT. In Proceedings of the 12th European Conference on Computer Systems (EuroSys '17).

[4] Philip A Bernstein, Vassos Hadzilacos, and Nathan Goodman. 1987. Concurrency Control and Recovery in Database Systems (1 ed.). Addison-Wesley.

[5] Dan Boneh, Amit Sahai, and Brent Waters. 2012. Functional Encryption: A New Vision for Public-Key Cryptography. Commun. ACM 55, 11 (Nov. 2012), 56-64.

[6] Mic Bowman, Andrea Miele, Michael Steiner, and Bruno Vavala. 2018. Private Data Objects: an Overview. arXiv:1807.05686. Retrieved from https://arxiv.org/ abs/1807.05686.

[7] Marcus Brandenburger, Christian Cachin, Matthias Lorenz, and Rüdiger Kapitza. 2017. Rollback and Forking Detection for Trusted Execution Environments using Lightweight Collective Memory. In Proceedings of the 47th Annual IEEE/IFIP International Conference on Dependable Systems and Networks (DSN '17).

[8] Jo Van Bulck, Marina Minkin, Ofir Weisse, Daniel Genkin, Baris Kasikci, Frank Piessens, Mark Silberstein, Thomas F. Wenisch, Yuval Yarom, and Raoul Strackx. 2018. Foreshadow: Extracting the Keys to the Intel SGX Kingdom with Transient Out-of-Order Execution. In Proceedings of the 27th USENIX Conference on Security Symposium (SEC '18).

[9] Miguel Castro and Barbara Liskov. 1999. Practical Byzantine Fault Tolerance. In Proceedings of the Third Symposium on Operating Systems Design and Implementation (OSDI'99).

[10] Julien Cathalo, Benoit Libert, and Jean-Jacques Quisquater. 2005. Efficient and Non-Interactive Timed-Release Encryption. In Proceedings of the 2005 International Conference on Information and Communications Security (ICICS '05).

[11] Raymond Cheng, Fan Zhang, Jernej Kos, Warren He, Nicholas Hynes, Noah M. Johnson, Ari Juels, Andrew Miller, and Dawn Song. 2019. Ekiden: A Platform for Confidentiality-Preserving, Trustworthy, and Performant Smart Contracts. In Proceedings of the 2019 IEEE European Symposium on Security and Privacy (EuroS\&P '19).

[12] Intel Corporation. 2021. Attestation Services for Intel SGX. https: //software.intel.com/content/www/us/en/develop/topics/software-guardextensions/attestation-services.html. Last accessed: 2021-09-15.

[13] Intel Corporation. 2021. Intel Attestation Service for SGX: API Documentation. https://api.trustedservices.intel.com/documents/sgx-attestation-api-spec. pdf. Last accessed: 2021-09-15.

[14] Intel Corporation. 2021. Intel SGX DCAP Orientation Guide. https: //software.intel.com/content/dam/develop/public/us/en/documents/intel-sgxdcap-ecdsa-orientation.pdf. Last accessed: 2021-09-15.

[15] Victor Costan, Ilia Lebedev, and Srinivas Devadas. 2016. Sanctum: Minimal Hardware Extensions for Strong Software Isolation. In Proceedings of the 25th USENIX Conference on Security Symposium (SEC '16).

[16] Hung Dang, Tien Tuan Anh Dinh, Ee-Chien Chang, and Beng Chin Ooi. 2017. Privacy-Preserving Computation with Trusted Computing via Scramble-thenCompute. In Proceedings of the 17th Privacy Enhancing Technologies Symposium (PETS '17).

[17] Hung Dang, Tien Tuan Anh Dinh, Dumitrel Loghin, Ee-Chien Chang, Qian Lin, and Beng Chin Ooi. 2019. Towards Scaling Blockchain Systems via Sharding. In Proceedings of the 2019 International Conference on Management of Data (SIGMOD '19).

[18] Cynthia Dwork and Aaron Roth. 2014. The Algorithmic Foundations of Differential Privacy. Found. Trends Theor. Comput. Sci. 9, 3-4 (Aug. 2014), 211-407.

[19] Facebook, Inc. 2021. Facebook Stories. https://www.facebook.com/help/ 862926927385914/?helpref=hc_fnav. Last accessed: 2021-09-15.

[20] Ben Fisch, Dhinakaran Vinayagamurthy, Dan Boneh, and Sergey Gorbunov. 2017. Iron: Functional Encryption using Intel SGX. In Proceedings of the 2017 ACM SIGSAC Conference on Computer and Communications Security (CCS '17).

[21] Michael J. Fischer, Nancy A. Lynch, and Michael S. Paterson. 1985. Impossibility of Distributed Consensus with One Faulty Process. F. ACM 32, 2 (April 1985), 374-382.

[22] Timothy Fraser, Lee Badger, and Mark Feldman. 1999. Hardening COTS Software with Generic Software Wrappers. In Proceedings of the 1999 IEEE Symposium on Security and Privacy (S\&P '99).

[23] Roxana Geambasu, Tadayoshi Kohno, Amit A. Levy, and Henry M. Levy. 2009. Vanish: Increasing Data Privacy with Self-Destructing Data. In Proceedings of the 18th Conference on USENIX Security Symposium (SEC '09).

[24] Foster D Hinshaw, Craig S Harris, and Sunil K Sarin. 2006. Distributed Concurrency Control using Serialization Ordering. US Patent 7146366. Retrieved from https://www.freepatentsonline.com/7146366.html

[25] Jim Isaak and Mina J. Hanna. 2018. User Data Privacy: Facebook, Cambridge Analytica, and Privacy Protection. Computer 51, 8 (Aug. 2018), 56-59.

[26] Jonathan Katz and Yehuda Lindell. 2014. Introduction to Modern Cryptography (2 ed.). Chapman and Hall/CRC.

[27] Dayeol Lee, David Kohlbrenner, Shweta Shinde, Krste Asanovic, and Dawn Song. 2020. Keystone: An Open Framework for Architecting Trusted Execution Environments. In Proceedings of the 15th European Conference on Computer Systems (EuroSys '20)

[28] Chao Li and Balaji Palanisamy. 2017. Timed-Release of Self-Emerging Data using Distributed Hash Tables. In Proceedings of the 37th IEEE International Conference on Distributed Computing Systems (ICDCS '17).

[29] WhatApp LLC. 2021. WhatsApp Disappearing Messages. https://faq.whatsapp. com/general/chats/about-disappearing-messages. Last accessed: 2021-09-15.

[30] Zohar Manna and Richard Waldinger. 1980. A Deductive Approach to Program Synthesis. ACM Trans. Program. Lang. Syst. 2, 1 (Jan. 1980), 90-121.

[31] Sai Krishna Deepak Maram, Fan Zhang, Lun Wang, Andrew Low, Yupeng Zhang, Ari Juels, and Dawn Song. 2019. CHURP: Dynamic-Committee Proactive Secret Sharing. In Proceedings of the 2019 ACM SIGSAC Conference on Computer and Communications Security (CCS '19).

[32] Sinisa Matetic, Mansoor Ahmed, Kari Kostiainen, Aritra Dhar, David M. Sommer, Arthur Gervais, Ari Juels, and Srdjan Capkun. 2017. ROTE: Rollback Protection for Trusted Execution. In Proceedings of the 26th Conference on USENIX Security Symposium (SEC '17).

[33] Frank McKeen, Ilya Alexandrovich, Alex Berenzon, Carlos V. Rozas, Hisham Shafi, Vedvyas Shanbhogue, and Uday R. Savagaonkar. 2013. Innovative Instructions and Software Model for Isolated Execution. In Proceedings of the 2nd International Workshop on Hardware and Architectural Support for Security and Privacy (HASP '13).

[34] Jianting Ning, Hung Dang, Ruomu Hou, and Ee-Chien Chang. 2018. Keeping Time-Release Secrets through Smart Contracts. Cryptology ePrint Archive, Report 2018/1166. Retrieved from https://ia.cr/2018/1166.

[35] Singapore Ministry of Communications and Information. 2019. Public Report of the Committee of the Inquiry into the Cyber Attack on SingHealth. https://www.mci.gov.sg/pressroom/news-and-stories/pressroom/ 2019/1/public-report-of-the-coi. Last accessed: 2021-09-15.

[36] The International Consortium of Investigative Journalists. 2021. The Panama Papers: Exposing the Rogue Offshore Finance Industry. https://www.icij.org/ investigations/panama-papers. Last accessed: 2021-09-15.

[37] Oleksii Oleksenko, Bohdan Trach, Robert Krahn, Mark Silberstein, and Christof Fetzer. 2018. Varys: Protecting SGX Enclaves from Practical Side-Channel Attacks. In Proceedings of the 2018 USENIX Annual Technical Conference (ATC '18).

[38] Diego Ongaro and John K Ousterhout. 2014. In Search of an Understandable Consensus Algorithm. In Proceedings of the 2014 USENIX Annual Technical Conference (ATC '14).

[39] Fred B. Schneider. 1990. Implementing Fault-Tolerant Services using the State Machine Approach: A Tutorial. ACM Comput. Surv. 22, 4 (Dec. 1990), 299-319.

[40] Adi Shamir. 1979. How to Share a Secret. Commun. ACM 22, 11 (Nov. 1979), 612-613.

[41] Dale Skeen. 1981. Nonblocking Commit Protocols. In Proceedings of the 1981 International Conference on Management of Data (SIGMOD '81).

[42] Martin R. Stytz. 2004. Considering Defense in Depth for Software Applications. IEEE Secur. Priv. 2, 1 (Jan. 2004).

[43] Pramod Subramanyan, Rohit Sinha, Ilia A. Lebedev, Srinivas Devadas, and Sanjit A. Seshia. 2017. A Formal Foundation for Secure Remote Execution of Enclaves. In Proceedings of the 2017 ACM SIGSAC Conference on Computer and Communications Security (CCS '17).

[44] Florian Tramèr, Fan Zhang, Huang Lin, Jean-Pierre Hubaux, Ari Juels, and Elaine Shi. 2017. Sealed-Glass Proofs: Using Transparent Enclaves to Prove and Sell Knowledge. In Proceedings of the 2017 IEEE European Symposium on Security and Privacy (EuroS\&P '17).

[45] European Union. 2016. General Data Protection Regulation, Article 17: Right to erasure ('right to be forgotten'). https://gdpr.eu/article-17-right-to-be-forgotten. Last accessed: 2021-09-15

[46] Moshe Y. Vardi and Pierre Wolper. 1986. An Automata-Theoretic Approach to Automatic Program Verification. In Proceedings of the First Symposium on Logic in Computer Science (LICS '86).

[47] Luis Vargas, Gyan Hazarika, Rachel Culpepper, Kevin R.B. Butler, Thomas Shrimpton, Doug Szajda, and Patrick Traynor. 2018. Mitigating Risk While Complying with Data Retention Laws. In Proceedings of the 2018 ACM SIGSAC Conference on Computer and Communications Security (CCS '18).

[48] Jinbo Xiong, Fenghua Li, Jianfeng Ma, Ximeng Liu, Zhiqiang Yao, and Patrick S. Chen. 2015. A Full Lifecycle Privacy Protection Scheme for Sensitive Data in Cloud Computing. Peer-to-Peer Networking and Applications 8, 6 (Nov. 2015), 1025-1037.

[49] Yuanzhong Xu, Weidong Cui, and Marcus Peinado. 2015. Controlled-Channel Attacks: Deterministic Side Channels for Untrusted Operating Systems. In Proceedings of the 2015 IEEE Symposium on Security and Privacy (S\&P '15). 